\title{
COMBINATION TREATMENT OF ACNE VULGARIS WITH 630 NM LIGHT EMITTING DIODE THERAPY
}

\author{
*Takafumi Ohshiro M.D. ${ }^{1}$, Toshio Ohshiro M.D., Ph.D. ${ }^{2}$, Katsumi Sasaki M.D. ${ }^{1}$, \\ Shunji Fujii M.D. ${ }^{1}$, and Yuki Taniguchi M.D. ${ }^{1}$ \\ 1: Obshiro Clinic, Tokyo, Japan \\ 2: Japan Medical Laser Laboratory, Tokyo, Japan
}

\begin{abstract}
Acne vulgaris is the most common skin disorder in adolescence. Various treatment methods, such as topical/oral antibiotics, topical retinoids, chemical peels and phototherapy, have been reported with various success rates. Recently the resistance to antibiotics necessitates the physician to seek novel treatment modalities. Since 2005, we have applied a $630 \mathrm{~nm}$ light emitting diode (LED)based device for acne in addition to conventional treatments. The conventional treatments, consisting of $\mathrm{CO}_{2}$ laser irradiation, dye laser irradiation, topical retinoids and topical/oral antibiotics, were performed for infectious lesions, and LED therapy was concomitantly performed once a week. Within 3 months, severe symptoms were improved and well controlled in all cases. The $630 \mathrm{~nm}$ LED irradiation seems to be an optional treatment in controlling the various grades of acne vulgaris.
\end{abstract}

Key Words: acne vulgaris, Propionibacterium acnes, endogenous porphyrins, light emitting diodes (LEDs), $630 \mathrm{~nm}$ combination treatment,

\section{Introduction}

Acne vulgaris is one of the most common skin conditions in dermatology. Topical and systemic antibiotics, retinoids and chemical-peelings have been used conventionally for acne treatment with variable success rates ${ }^{(1-4)}$. However, a recent increase in the resistance to antibiotics of Propionibacterium acnes (P. acnes) and adverse effects of oral antibiotics are becoming obstacles to acne treatment, thus necessitating physicians to seek novel treatment modalities.

Recently, various forms of phototherapy for active acne have been attracting attention and a number of methodologies have been reported. It has been revealed that various wavelengths of visible light are capable of activating the endogenous porphyrins of $P$. acnes, which results in a photochemical reaction to

\section{Addressee for Correspondence:}

Corresponding Author

Takafumi Ohshiro M.D.

JR Shinanomachi-building 2F, 34 Shinanomachi, Shinjuku-

ku, Tokyo, Japan

160-0016

TEL: +81-3-3352-0046

FAX: +81-3-3354-1103

E-mail: ttoo@ohshiro.com destroy the bacterium through the creation of cytocidal singlet oxygen and other free radicals. The absorption

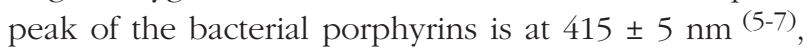
so LED-based systems emitting at or around $415 \mathrm{~nm}$ (5) have been widely used. But the blue light therapy is not popular in Asians, because of insufficient penetration depth to destroy sebaceous gland due to light absorption by melanin in the epidermis and the possibility of epidermal melanocyte stimulation leading to darkening resembling secondary hyperpigmentation. In addition, photodynamic therapy (PDT) with exogenous 20\% 5-Aminolevulinic acid (5-ALA) and $630 \mathrm{~nm}$ lasers or LED systems has been applied for acne, and promising results have been reported (6,7). However post inflammatory hyperpigmentation after PDT with topical 5-ALA is not acceptable, and that is once again the problem with the Asian skin phenotype when the ALA percentage is too high, or the ALA is over-activated.

In 2004, we developed a novel $630 \mathrm{~nm}$ light emitting diode (LED) device with convectional cooling system for the treatment of photoaged skin and various inflammatory lesions. Since 2005, we have applied this system in the treatment of acne vulgaris. As a result,

Manuscript received: July 2007

Accepted for publication: August 2007 
Table 1: The Grading Criteria of Acne Severity Defined by Burton et al.

\begin{tabular}{ll}
\hline Grade & Type of lesions \\
\hline Grade 0 & No acne lesions \\
Grade 1 & $\begin{array}{l}\text { Sub-clinical acne: a few insignificant comedones } \\
\text { which can be seen only on careful inspection }\end{array}$ \\
Grade 2 & $\begin{array}{l}\text { Mild acne: a few comedones and a few small } \\
\text { papules or pustules are seen }\end{array}$ \\
Grade 3 & $\begin{array}{l}\text { Moderate acne: Prompt papules/pustules are easi- } \\
\text { ly recognized }\end{array}$ \\
Grade 4 & $\begin{array}{l}\text { Severe acne: Cysts are often found } \\
\text { Grade 5 }\end{array}$ \\
$\begin{array}{l}\text { Extremely severe acne: Widespread inflammatory } \\
\text { lesions and manny large pustules or cysts are found }\end{array}$ \\
\hline
\end{tabular}

the combination therapy with conventional treatments and $630 \mathrm{~nm}$ LED therapy was found to be beneficial and useful to control the condition of acne vulgaris.

\section{Materials and Methods}

\section{LED source}

The LED system used for this report was the Photorefresher ${ }^{\circledR}$ (Japan Medical Laser Laboratory, Tokyo, Japan). The system consists of single curved panel, which can cover the whole human face, containing 4800 LEDs with a convectional cooling system. The panel of the system delivers symmetrical peak wavelengths $(641 \pm 18 \mathrm{~nm})$ within an irradiation field of $3080 \mathrm{~cm}^{2}$. The dominant wavelength of each LED is 630 $\mathrm{nm}$.

The power density and energy desnity/dose were set $3.3 \mathrm{~mW} / \mathrm{cm}^{2}$ and $80 \mathrm{~J} / \mathrm{cm}^{2}$, respectively, for each treatment.

\section{Treatment methods}

We have used various methods according to the severity of acne. The Burton Scale (4) (Table 1) is useful to diagnose the acne condition. Our conventional treatment methods are as shown in Table 2.

Under the reviewed regime, including $630 \mathrm{~nm}$ LED therapy, ointment is used twice a day. Lasers were used once or twice a month. During the treatment period, $630 \mathrm{~nm}$ LED therapy was used every 1 or 2 weeks.
Table 2: Conventional treatment regimens for acne vulgaris shown by Burton grade

\begin{tabular}{ll}
\hline Grade & Treatment \\
\hline Grades 1 \& 2 & $\begin{array}{l}\text { Topical retinoids }(0.025 \%) \\
\text { CO2 laser for drainage of secretion } \\
\text { Pulsed dye laser irradiation for mild inflam- } \\
\text { mation }\end{array}$ \\
\hline Grade 3 & $\begin{array}{l}\text { Topical retinoids }(0.025,0.05 \%) \\
\text { Topical antibiotics (1\% Clyndamycin gel) } \\
\text { CO2 laser for drainage of bacteria } \\
\text { Pulsed dye laser for resolution of erythema }\end{array}$ \\
\hline Grades 4 \&5 & $\begin{array}{l}\text { topical retinoids (0.05\%) and oral \& topical } \\
\text { antibiotics } \\
\text { CO2 laser and pulsed dye laser }\end{array}$ \\
\hline
\end{tabular}

The clinical photographs and the Visia II system (Canfield Scientifics Inc., NJ, USA) were used to assess the subjects. The indirect measurement of P. acnes fluorescence on skin captured with the Visia system, comparing pretreatment baseline measurements with post-treatment findings, was used as a marker to assess the suppression of bacterial porphyrin fluorescence by the treatments, and hence the decreased $P$. acnes count.

\section{Case Reports}

We present our representative cases.

\section{Case 1: Burton Grade 5 [Fig.1-2]}

A 15-year-old Japanese male had suffered from resistant prominent papules and comedones. (Burton Grade 5) He has previously been treated with oral antibiotics and chemical peeling with glycolic acid.

At the first visit to our clinic, drainage for comedones using the $\mathrm{CO} 2$ laser, and pulsed dye laser irradiation for the inflammatory component of the papules were performed. Topical retinoids $(0.025 \%)$ twice a day was started. Within 2 months the symptoms were was almost cleared, but the condition gradually deteriorated when only conventional treatments were used for follow up. In order to maintain his good results, the patient has continued the combination treatment with LED therapy once or twice a month, and the results have been very good. 


\section{Case 2: Burton Grade 4 [Fig.3]}

A 22-year-old Japanese female(Burton Grade 4) presented with facial acne which had been resistant to various treatments such as oral antibiotics, chemical peeling with glycolic acid and Chinese herbal treatment. The conventional treatments had improved the severe pustules and comedones, but prolonged erythema of the inflammatory papules was still present. Combination treatment with LED therapy once a week improved the symptoms within 3 months.

\section{Case 3: Burton Grade 3 [Fig.4]}

A 26-year-old Japanese female had suffered from intractable acne vulgaris lesions (Burton Grade 3) on her face for the 10 years before presenting at our clinic. The appearance of new lesions coincided with her menstrual cycle and the acne was resistant to oral antibiotics and chemical peeling with glycolic acid.

At first, she was treated with our conventional treatments with topical retinoids $(0.025 \%)$, topical antibiotics, drainage with $\mathrm{CO} 2$ laser, and pulsed dye laser treatment for inflammatory lesions. The severe facial papules and pustules were easily controlled, but prolonged inflammatory erythema could not be improved. The combination of LED therapy was started once a week, and the symptoms regressed in three months. After improvement, the interval of LED therapy was gradually extended and now her acne is well controlled with one LED therapy session per month.

\section{Case 4: Burton Grade 3 [Fig.5]}

A 15-year-old Japanese female (Burton Grade 2) had been treated with oral and topical antibiotics and chemical peeling in another dermatological clinic. In our clinic topical retinoids (0.025\%) and LED therapy once week was started. The inflammatory lesions were remarkably improved within 3 months.

\section{Discussion}

Acne vulgaris is a chronic inflammatory disease of the pilosebaceous units in adolescents and young adults aged 15-25 years, although there is still a one-in-two chance of contracting acne even after the mid-20's. The increased sebum secretion and the abnormal desquamation of the follicular epithelium lead to obstruction of the pilosebaceous canal and cause comedones formation. P. acnes migrating from the stratum corneum into the trapped sebum plays an important role in mediating follicular inflammation. Various factors, including hormonal disorders, psychological stress and dietetic problems, are associated with the aggravation of acne. Various treatments, such as oral/topical antibiotics, topical retinoids, lasers and photodynamic therapy, have been reported. The mode of treatment must be chosen according to the severity and grading of the acne,

We have applied our own combination methods for acne using topical retinoids, topical antibiotics, $\mathrm{CO} 2$ laser and pulsed dye laser. Concerning comedone formation, topical retinoids are effective for suppression of the hyperkeratosis of the pilosebaceous units and desquamation of the hair follicular plugs. For prevention of the inflammatory papules and pustules, the drainage of pus using the focused beam of the $\mathrm{CO}_{2}$ laser and the reduction of redness using the pulsed dye laser is useful. Only in cases where infectious signs such as severe pustules are seen, oral and topical antibiotics are given. We are worried that routine use of antibiotics to control the P. acnes may cause drug resistance in this bacterium, and we therefore use them in limited cases.

On the contrary, in phototherapy for acne, 415 nm LED therapy has attracted attention (5). Acute inflammatory acne lesions contain $P$. acnes which produces endogenous porphyrins, protoporphyrin IX (Pp IX) and coproporphyrin III (Cp III). Both of these porphyrins have a strong absorption peak at around 415 $\mathrm{nm}$ in the Soret waveband. The irradiation of a $415 \mathrm{~nm}$ blue light will photochemically generate singlet oxygen within $P$. acnes which results in a process known as endogenous photodynamic therapy ${ }^{(8)}$. But $415 \mathrm{~nm}$ LED on its own for acne vulgaris is not popular in Asians. The penetration depth of the blue light is not enough to reach sufficient $P$. acnes deep in the sebaceous gland, mainly because of absorption by a competing chromophore, mainly melanin, in the epidermis. The insufficient penetration depth brings about a poor response in cases of severe acne. Recently photodynamic therapy (PDT) with 20\% 5-Aminolevulinic acid (5-ALA) and $630 \mathrm{~nm}$ laser or light emitting diode has been applied for acne, and various good results have been reported. However the prolonged hyperphotosensitivity and post inflammatory hyperpigmentation after PDT which is a common side effect in the Asian skin type, is not acceptable except in severe cases. A recent report by Lee et al., however, showed that the combination of blue $415 \mathrm{~nm}$ with red $633 \mathrm{~nm}$, applied sequentially, achieved excellent results in the Asian skin type. (9)

Our conventional methods certainly improved the acute infectious phase as in Case 1, but it is difficult to improve the prolonged inflammatory papules within a short period of time. To treat the inflammatory lesions within a short time and to keep the patients symptoms- 


\section{ORIGINAL ARTICLES}
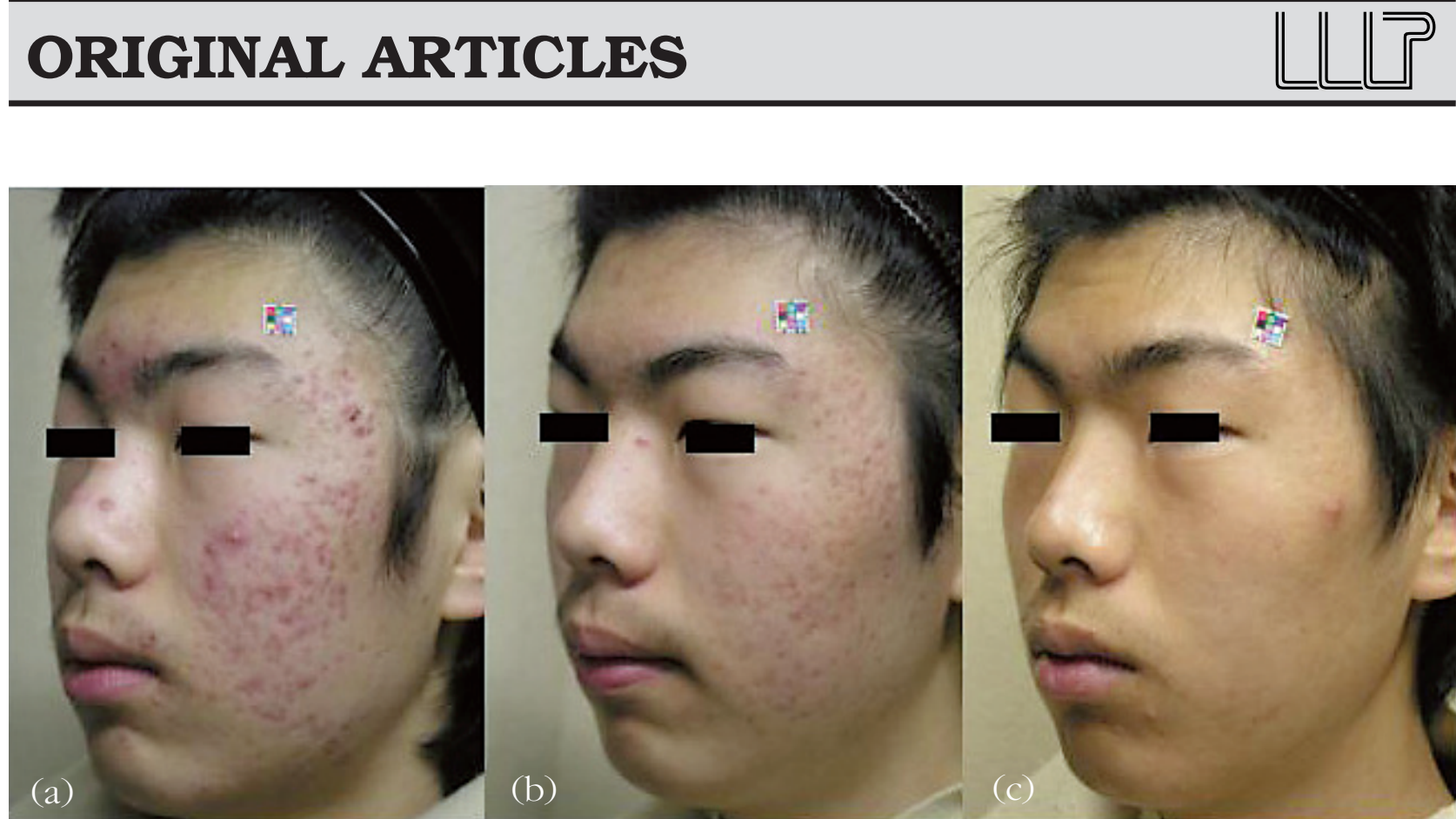

Fig. 1: 15-year-old Japanese male, with severe inflammatory acne (Burton Grade 5)

(a) Before treatment

(b) 2 months after the conventional treatments started

(c) 3 months after the additional $630 \mathrm{~nm}$ LED therapy
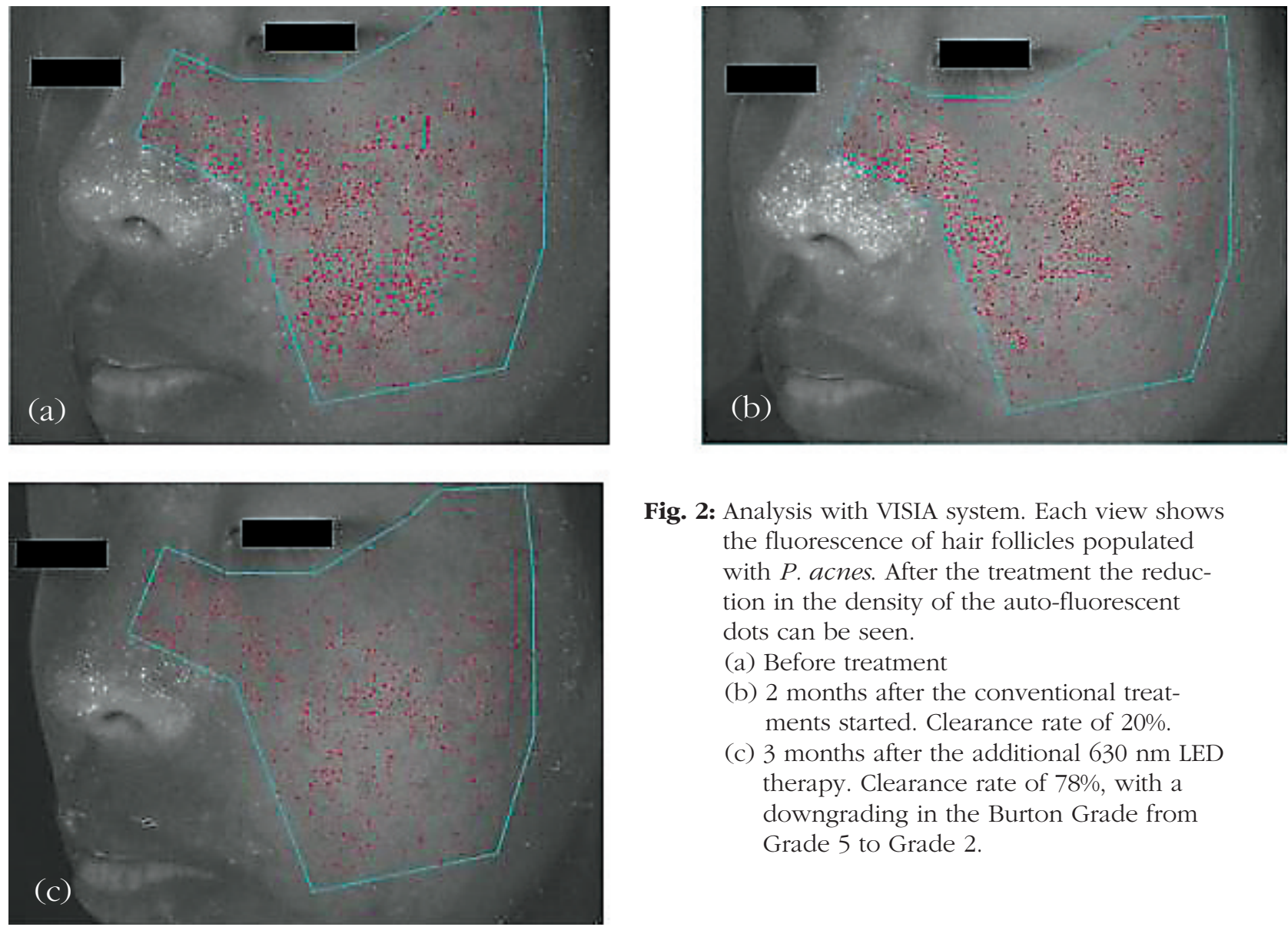

Fig. 2: Analysis with VISIA system. Each view shows the fluorescence of hair follicles populated with $P$. acnes. After the treatment the reduction in the density of the auto-fluorescent dots can be seen.

(a) Before treatment

(b) 2 months after the conventional treatments started. Clearance rate of $20 \%$.

(c) 3 months after the additional $630 \mathrm{~nm}$ LED therapy. Clearance rate of $78 \%$, with a downgrading in the Burton Grade from Grade 5 to Grade 2. 


แLU? ORIGINAL ARTICLES

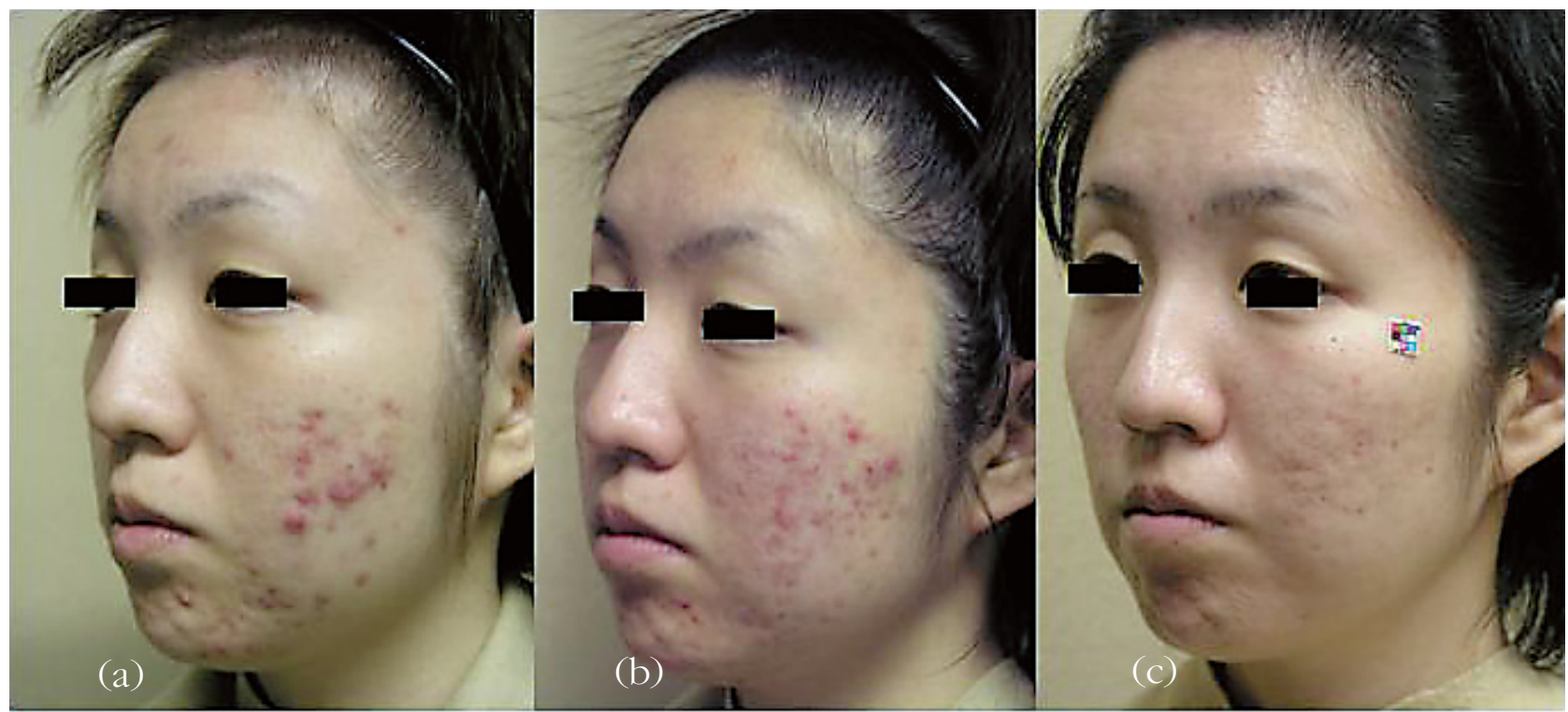

Fig. 3: 22-year-old Japanese female, with severe acne (Burton Grade 4)

(a) Before treatment

(b) 3 months after the conventional treatments started.

(c) 3 months after the additional $630 \mathrm{~nm}$ LED therapy. Remarkable improvement was obtained with downshift to Grade 2.
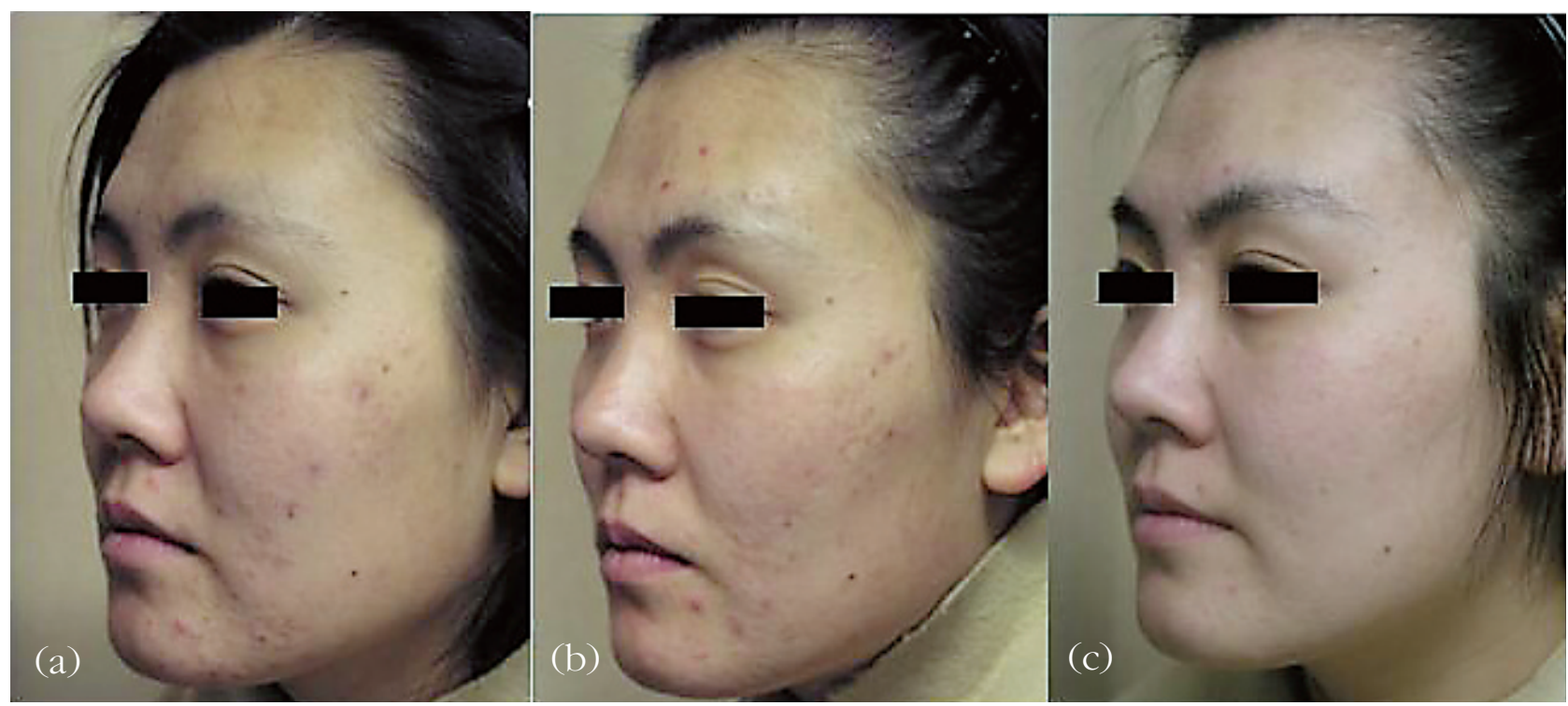

Fig. 4: 26-year-old Japanese female, with moderate acne (Burton Grade 3)

(a) Before treatment

(b) 2 months after the conventional treatments started.

(c) 3 months after the additional $630 \mathrm{~nm}$ LED therapy. Burton scale improved to Grade 1. 


\section{ORIGINAL ARTICLES}

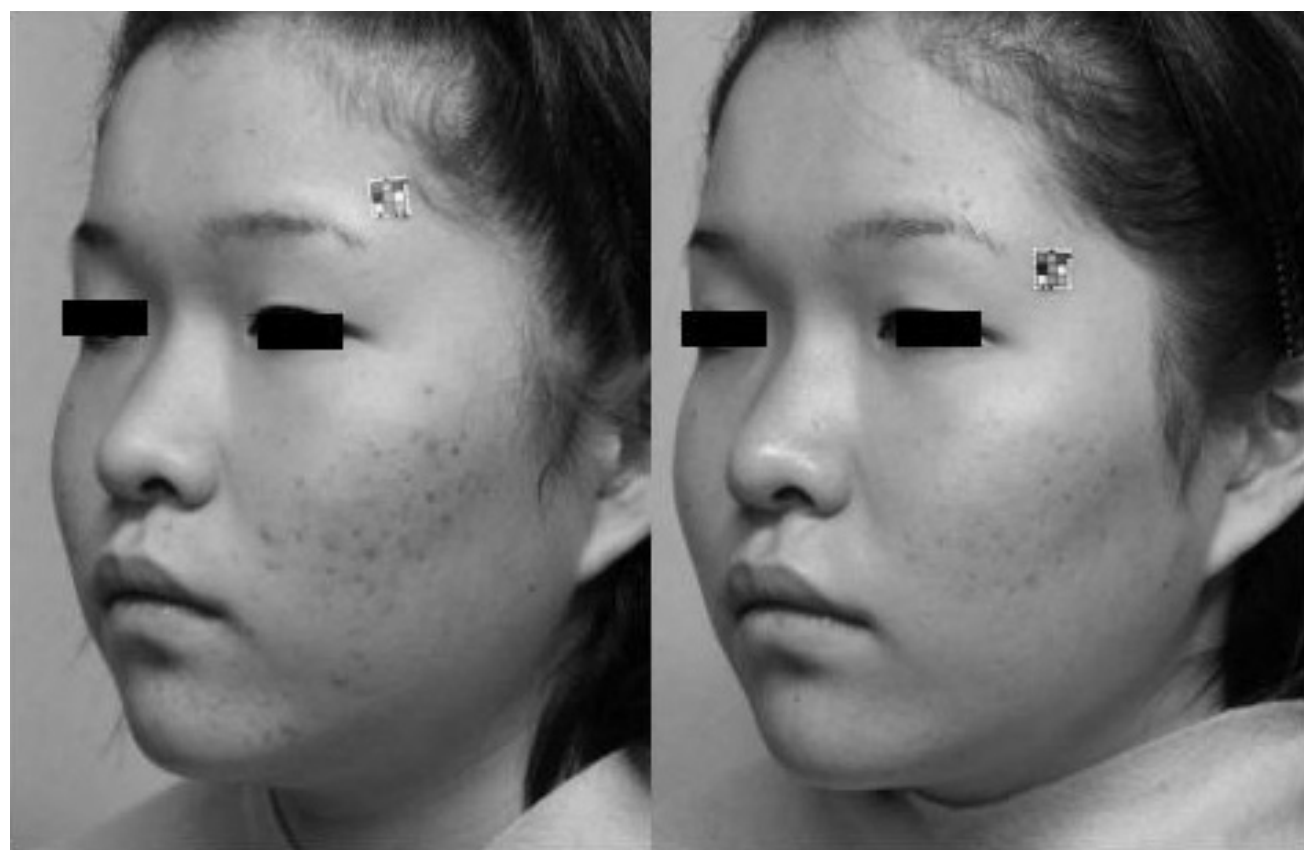

Fig. 5: 15-year-old Japanese female with moderate acne (Burton Grade 3)

(a) Before treatment

(b) 3 months after the combination treatment with $630 \mathrm{~nm}$ LED. A downgrading to Burton Grade 1 has been achieved.

free, we have applied a $630 \mathrm{~nm}$ LED system. In combination cases, the improvements were obtained by a treatment of once a week for 3 months. To maintain the good condition, the interval of the treatment was gradually extended to once a month.

The $630 \mathrm{~nm}$ LED has a deeper penetration depth in the skin, because of longer wavelength and less absorption in competing chromophores such as hemoglobin and melanin, at that wavelength Irradiation of tissue with wavelengths around $630 \mathrm{~nm}$ has been reported to lead to an anti-inflammatory reaction through enhancement of local blood flow, and control of the inflammatory phase cells such as mast cells and macrophages though photoactivation of the respiratory chain of the mitochondria (8). The same basic photoreaction will also activate fibroblasts to help heal the dermal wound created by the acne lesion. We think these particular characteristics of this wavelength might achieve the anti-inflammatory effect for acne. In addition, the endogenous porphyrins, Pp IX and Cp III, have various absorption peaks, the maximum around $415 \mathrm{~nm}$, but with small peaks at 509, 544, 584 and 635 $\mathrm{nm}$ for Pp IX ${ }^{(5)}$. We consider the small peak in the red waveband might play an important role. Irradiation of Pp IX with $630 \mathrm{~nm}$ LED light, in addition to its known anti-inflammatory and wound healing properties, might also slightly activate the porphyrin and induce a moderate endogenous photodynamic reaction via production of singlet oxygen having a cytotoxic effect on the $P$. acnes. If this were the case, the combination of the anti-inflammatory effect and the photodynamic reaction could achieve improvement of the inflammation associated with severe acne vulgaris.

For the treatment of acne vulgaris, it is important to improve the various stages of acne and to maintain good improvements in the facial condition once they have been achieved. Even if an appropriate treatment is done according to grade of the individual condition, there is no meaning if it cannot be controlled in a symptom-free state over the post-treatment period. Our combination treatment method for acne was easy to deliver, and side effect free. Further investigation and long term follow up are required, but it would appear to be an interesting treatment option for acne vulgaris. 


\section{References}

1. Leyden JJ (1997): Therapy for acne vulgaris. New England Journal of Medicine, 336:1156-1162

2. Haidar A and Shaw CC (2004): Treatment of acne vulgaris. Journal of the American Medical Association, 292:726-735

3. Berson DS, Chalker DK, Harper JC, Leyden JJ, Shalita AR and Webster GF (2003): Current concepts in the treatment of acne: report from a clinical roundtable. Cutis, 72:5-13

4. Burton JL, Cunliffe WJ, Stafford I and Shuster S (1971): The prevalence of acne vulgaris in adolescence. British Journal of Dermatology, 85:119-126

5. Kjeldstad B, Johnsson A (1986): An action spectrum for blue and near ultraviolet inactivation of Propionibacterium acnes; with emphasis on a possible porphyrin photosensitization. Journal of Photochemistry and Photobiology, 43:67-70

6. Mills OH, Kligman AM (1978): Ultraviolet phototherapy and photochemotherapy of acne vulgaris. Archives of Dermatology, 114:221-223

7. Sigurdsson V, Knulst AC, van Weelden H (1997). Phototherapy of acne vulgaris with visible light. Dermatology, 194:256-260

8. Touma DJ and Gilchrest BA (2003): Topical photo- dynamic therapy: a new tool in cosmetic dermatology. Seminars in Cutaneous Medicine and Surgery, 22:124-130

9. Itoh Y, Ninomiya Y, Tajima S and Ishibashi A (2000): Photodynamic therapy for acne vulgaris with topical 5-aminolevulinic acid. Archives of Dermatology, 136:1093-1095

10. Itoh Y, Ninomiya Y, Tajima S and Isibashi A (2001): Photodynamic therapy of acne vulgaris with topical delta-aminolaevulinic acid and incoherent light in Japanese patients. British Journal of Dermatology, 144:575-579

11. Calderhead RG (2007): The photobiological basics behind light-emitting diode (LED) phototherapy. Laser Therapy, 16: 97-108.

12. Lee SY, You CE, and Park MY (2007): Blue and red light combination LED phototherapy for acne vulgaris in patients with skin phototype IV. Lasers Surg Med., 39: 180-188.

13. Karu T (1999): Primary and secondary mechanisms of action of visible to near-IR radiation on cells. Journal of Photochemistry and Photobiology B, 49:1-17 